\title{
Sleep Stage Classification by a Combination of Actigraphic and Heart Rate Signals
}

\author{
Emi Yuda ${ }^{1}$, Yutaka Yoshida ${ }^{1}$, Ryujiro Sasanabe ${ }^{2}$, Haruhito Tanaka ${ }^{3}$, Toshiaki Shiomi ${ }^{2}$ \\ and Junichiro Hayano ${ }^{1, *}$ \\ 1 Graduate School of Medical Sciences, Nagoya City University, Nagoya 467-8602, Japan; \\ emi21@med.nagoya-cu.ac.jp (E.Y.); yyoshida@med.nagoya-cu.ac.jp (Y.Y.) \\ 2 Sleep Disorder Center, Aichi Medical University Hospital, Nagakute 480-1195, Japan; \\ sasanabe@aichi-med-u.ac.jp (R.S.); toshiaki@aichi-med-u.ac.jp (T.S.) \\ 3 Gifu Mates Sleep Clinic, Gifu 500-8384, Japan; hata0507@gmail.com \\ * Correspondence: hayano@med.nagoya-cu.ac.jp; Tel.: +81-52-853-8507
}

Received: 25 September 2017; Accepted: 9 November 2017; Published: 13 November 2017

\begin{abstract}
Although heart rate variability and actigraphic data have been used for sleep-wake or sleep stage classifications, there are few studies on the combined use of them. Recent wearable sensors, however, equip both pulse wave and actigraphic sensors. This paper presents results on the performance of sleep stage classification by a combination of heart rate and actigraphic signals. We studied 40,643 epochs (length $3 \mathrm{~min}$ ) of polysomnographic data in 289 subjects. A combined model, consisting of autonomic functional indices from heart rate variability and body movement indices derived from actigraphic data, discriminated non-rapid-eye-movement (REM) sleep from waking/REM sleep with 76.9\% sensitivity, $74.5 \%$ specificity, $75.8 \%$ accuracy, and a Cohen's kappa of 0.514 . The combination was also useful for discriminating between REM sleep and waking at $77.2 \%$ sensitivity, $72.3 \%$ specificity, $74.5 \%$ accuracy, and a kappa of 0.491 .
\end{abstract}

Keywords: actigraphy; heart rate variability; polysomnography; sleep; sleep stage

\section{Introduction}

With the widespread use of wearable sensors, heartbeat and physical activity data are accumulating and forming physiological big data. To utilize these data for self-healthcare, the detection of sleep period and assessment of sleep quality are important issues. Although plenty of earlier studies have reported various algorithms and methods for this purpose, most of them used either heartbeat or actigraphic data [1-8], and only a few of them reported the combined use of both parameters in a small population [9]. Because many recent wearable sensors of electrocardiogram (ECG)/pulse wave also equip triaxial accelerometers, algorithms utilizing both data modalities seem useful. In addition, the sample size of earlier studies was small compared to the number of features used for sleep stage classification. Thus, their performance when applying real world data from a large population is unknown.

In the present study, we collected ECG and actigraphic data during a polysomnographic study (PSG) in 289 subjects. We examined the performance of sleep stage classification by the combination of heart rate variability (HRV)-derived autonomic indices and actigraphic parameters by comparing it with sleep stage classification determined by PSG. Autonomic function state is thought to differ between non-rapid-eye-movement (REM) (NREM) sleep and waking/REM sleep [10-13], while it is similar between waking and REM sleep [8]. In contrast, the magnitude of body movement (BM) differs between waking and REM sleep due to its suppression during REM sleep. Thus, we evaluated the classification performances separately for the discrimination of NREM sleep from waking/REM sleep and of waking from REM sleep. 


\section{Methods}

The present study was performed according to the protocol that was approved by the Institutional Review Board of Nagoya City University Graduate School of Medical Sciences and Nagoya City University Hospital (No. 60160097).

\subsection{Data Collection}

We studied ECG and wrist actigraphic data recorded simultaneously with an overnight PSG in 289 subjects (199 males and 90 females, median age (interquartile range (IQR)) 52 (37-65) years). They were consecutive subjects whose ECG showed sinus rhythm. The PSG examinations were performed in the Sleep Disorder Center, Aichi Medical University Hospital (Nagakute city, Aichi, Japan) for 168 subjects and the Gifu Mates Sleep Clinic (Gifu city, Gifu, Japan) for 121 subjects. The subjects included 36 that were normal, 168 that had sleep apnea syndrome, 31 that had REM sleep behavioral disorder, 23 that had insomnia, 14 that had hypersomnia, 12 that had narcolepsy, and 5 that had restless leg syndrome. None of the subjects had heart diseases or atrial fibrillation.

The ECG was recorded with a modified V2 lead and sampled at $500 \mathrm{~Hz}$. Actigraphic data were collected by a wrist actigraphic sensor (HFM-3D Prototype, Suzuken Co., Nagoya, Japan), which digitized and stored triaxial acceleration data at $31.25 \mathrm{~Hz}$.

Sleep stages were scored per 30-s window according to the American Academy of Sleep Medicine (AASM)'s guidelines by registered polysomnographic technicians of each center. Then, six consecutive windows were grouped together into one epoch (length, $3 \mathrm{~min}$ ). A stage of the epoch was defined as those of the majority among six windows, i.e., if the stage of $>4$ windows was the same, it was chosen as a stage of the epoch. If there was no majority stage among six windows, the epoch was defined as a transitional stage. This exclusion criterion is a mitigated criterion used in earlier studies [1].

\subsection{Data Analysis}

The PSG, ECG, and actigraphic data were synchronized by entering a time marker into the PSG and ECG recording when the actigraphic recording was started. From the ECG data, all R waves were detected and labeled for the rhythm of beat (sinus, ventricular ectopic, supraventricular ectopic, noise, etc.) and R-R interval time series were generated. The R-R interval time series and actigraphic data were divided into consecutive 3-min epochs. For each epoch, heart rate variability (HRV) was analyzed by the methods that have been reported elsewhere [14]. Briefly, R-R intervals were interpolated by a step function only using consecutive sinus rhythm (normal-to-normal) R-R intervals (NN intervals). Then, interpolated data were resampled equidistantly in time, filtered with the Hanning window, and analyzed by fast Fourier transformation. After correcting for the losses of variance resulting from the Hanning window filtering, the obtained power spectra were integrated for the power of very low frequency (VLF, 0.003-0.04 Hz), low frequency (LF, 0.04-0.15 Hz), and high frequency (HF, 0.15-0.45 Hz) components. According to the guideline for heart rate variability analysis [15], the power of these components was transformed into logarithmic values. The ratio of LF to HF power (LF/HF) was also calculated.

For the actigraphic data in each 3-min epoch, acceleration signals in the $\mathrm{x}, \mathrm{y}$, and $\mathrm{z}$ axes were bandpass filtered $(0.02-0.08 \mathrm{~Hz})$ to remove baseline trend and high-frequency noise, and were composed into the magnitude, $A c t(t)$, with the following equation.

$$
\operatorname{Act}(t)=\sqrt{x(t)^{2}+y(t)^{2}+z(t)^{2}}
$$

Then, the average, median, maximum, and upper 95\% values of $A c t(t)$ during each 3-min epoch were calculated as the corresponding indices of BM, respectively. 


\subsection{Statistical Analysis}

We used the program package of Statistical Analysis System (SAS institute, Cary, NC, USA) for the statistical analyses. We evaluated the performance of classifications between NREM sleep and waking/REM sleep and between waking and REM sleep by multivariate logistic regression analyses, in which the heart rate (HR), VLF, LF, HF, LF/HF, and the average, median, maximum, and upper $95 \%$ values of $\mathrm{BM}$ were candidate independent variables and regression models were generated by stepwise variable selection. We evaluated the sensitivity and specificity of the classification with the area-under-curve (AUC) of a receiver-operating characteristic curve (ROC) analysis and using the best cutoff criteria, sensitivity, specificity, and accuracy (fraction of correctly classified) were calculated. We also computed Cohen's kappa statistics of agreement. Statistical significance was considered for $p<0.05$.

\section{Results}

A total of 40,643 epochs (length, $3 \mathrm{~min}$ ) were obtained (Table 1). After excluding the 12,337 with a transitional stage, 28,306 were used for analyses. Figure 1 shows the indices of HRV and BM by sleep stages. HR and VLF were smaller and HF was greater during the NREM stage. All indices of BM were greater during the waking stage.

Table 1. Number of studied epochs and their stages.

\begin{tabular}{ccc}
\hline Sleep Stage & Epoch (Length, $\mathbf{3}$ min) & $\mathbf{\%}$ \\
\hline Waking & 7173 & 17.6 \\
REM & 6049 & 14.9 \\
NREM & 15,084 & 37.0 \\
Transitional & 12,337 & 30.5 \\
Total & 40,643 & 100.0 \\
\hline
\end{tabular}

REM: rapid-eye-movement; NREM: non-rapid-eye-movement.
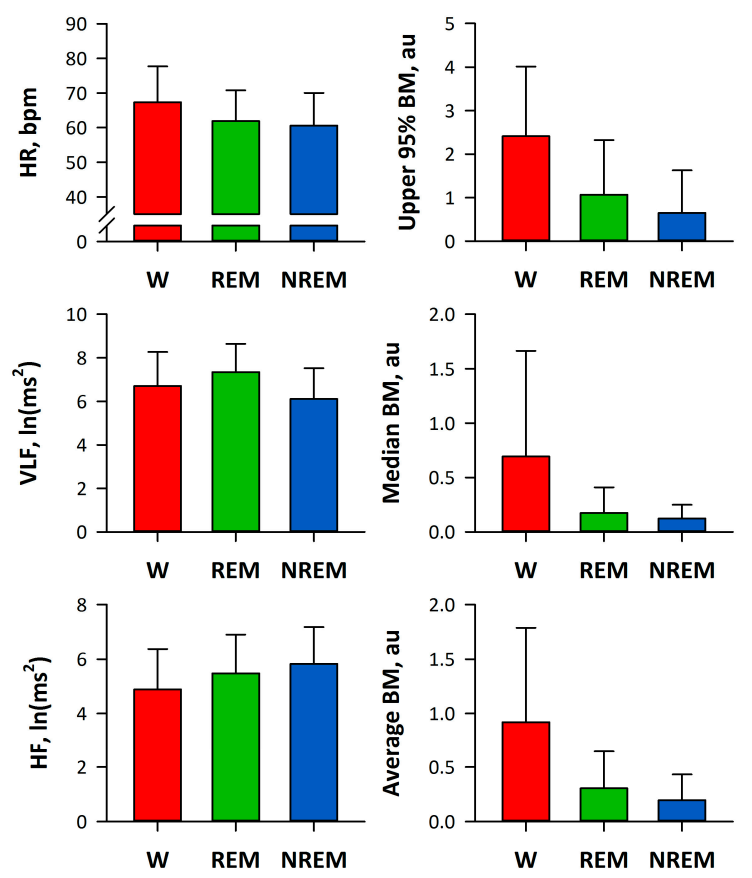

Figure 1. Sleep stages and indices of heart rate variability and body movement. W: waking; REM: rapid-eye-movement sleep; NREM: non-REM sleep; HF: high frequency; VLF: very low frequency; HR: heart rate; BM: body movement. 
The logistic regression analysis revealed that the NREM stage was discriminated from the waking and REM stages by upper 95\% BM, HF, VLF, median BM, and HR (Table 2) and that the REM stage was discriminated from the waking stage by upper $95 \%$ BM, VLF, average BM, and HR (Table 3). The ROC curve analysis showed that the models discriminate the NREM stage with an AUC of 0.830, 76.9\% sensitivity, $74.5 \%$ specificity, $75.8 \%$ accuracy, and a Cohen's kappa statistic of 0.514 and discriminate the REM stage with an AUC of $0.20,77.2 \%$ sensitivity, $72.3 \%$ specificity, $74.5 \%$ accuracy, and a kappa statistic of 0.491 (Table 4).

Table 2. Logistic model discriminating NREM sleep from waking and REM sleep.

\begin{tabular}{ccccc}
\hline & Coefficient & SE & Score $\chi^{2}$ & $p$ \\
\hline Upper 95\% BM & 0.19 & 0.01 & 4526 & $<0.0001$ \\
HF & -0.62 & 0.01 & 1121 & $<0.0001$ \\
VLF & 0.71 & 0.01 & 3155 & $<0.0001$ \\
Median BM & 2.06 & 0.13 & 429 & $<0.0001$ \\
HR & 0.03 & 0.002 & 280 & $<0.0001$ \\
\hline \multicolumn{5}{c}{ SE: standard error. }
\end{tabular}

Table 3. Logistic model discriminating waking from REM sleep.

\begin{tabular}{ccccc}
\hline & Coefficient & SE & Score $\chi^{2}$ & $p$ \\
\hline Upper 95\% BM & 0.21 & 0.03 & 2275 & $<0.0001$ \\
VLF & -0.52 & 0.02 & 1464 & $<0.0001$ \\
Average BM & 1.72 & 0.11 & 328 & $<0.0001$ \\
HR & 0.03 & 0.002 & 137 & $<0.0001$ \\
\hline
\end{tabular}

Table 4. Receiver-operating characteristic (ROC) curve analysis of sleep stage classification.

\begin{tabular}{ccc}
\hline & NREM from the Other Stages & REM from Waking \\
\hline AUC & $0.830(0.826-0.835)$ & $0.820(0.813-0.827)$ \\
Sensitivity, $\%$ & 76.9 & 77.2 \\
Specificity, $\%$ & 74.5 & 72.3 \\
Accuracy, $\%$ & 75.8 & 74.5 \\
Cohen's kappa & 0.514 & 0.491 \\
\hline \multicolumn{3}{c}{}
\end{tabular}

\section{Discussion}

In this study, we demonstrated the performance of sleep stage classification by the indices obtained from HR and actigraphic signals. We studied 40,643 epochs (length, 3 min) of PSG data in 289 subjects. We observed that the NREM stage was discriminated from the waking/REM stages by the combination of HF, VLF, HR, and BM indices with $75.8 \%$ accuracy and kappa $=0.514$, and that REM sleep was discriminated from waking by the combination of VLF, HR, and BM indices with $74.5 \%$ accuracy and kappa $=0.491$. These observations indicate that the combined use of autonomic functional indices from HRV and BM indices derived from actigraphic data are useful for discriminating the NREM stage from the REM/waking stages and for discriminating the REM stage from the waking stage.

Many earlier studies have reported the performance of sleep stage classification by HRV indices [1-3,8]. Penzel et al. [1] studied 78 subjects undergoing a PSG. They analyzed HRV indices (mean R-R interval, SD of R-R interval, VLF, LF, HF, and LF/HF) and scaling exponents for 5-min consecutive segments whose sleep stage was defined as that continued for $>3 \mathrm{~min}$ in the segment. They reported $85 \%$ accuracy for sleep stage separation by a discriminant model consisting of the mean and SD of R-R intervals and scaling exponents. Using 18 PSG data in the MIT/BIH Polysomnographic Database, Adnane et al. [2] reported 80\% accuracy and a Cohen's kappa of 0.41 for the classification 
of wake and sleep of 30-s epochs with 10 features of HRV and heart rate dynamics selected by a support vector machine recursive elimination system. Fonseca et al. [3], in a study of 48 PSG data in healthy adults, reported $80 \%$ accuracy and a kappa of 0.56 in the classification of 30-s epochs of wake, NREM sleep, and REM sleep with 80 features selected from 142 features of ECG and thoracic respiratory effort. Additionally, Aktaruzzaman et al. [8], in a study of 20 PSG data, reported $84 \%$ and $71 \%$ accuracy and kappa of 0.68 and 0.45 in the classification of 5-min segments for NREM versus REM and for sleep versus wakefulness, respectively, with 4 features selected from 12 HRV features. Although the discriminant accuracy that we observed in this study seems comparable to those reported in these earlier studies, it should be noted that, in these earlier studies, a greater number of HRV indices was used in a smaller sample size of subjects than ours. Thus, the classification models obtained in these previous studies have a higher risk of overfitting to the data used for the analysis, which could result in a lower performance when they are applied to new data from other groups of subjects.

There are also many earlier studies reporting on the sleep-wakefulness classification performance of BM assessed by actigraphy [4-6]. In a study of 41 subjects undergoing a PSG examination, Cole et al. [4] reported that sleep period was distinguished by a wrist actigraphy with $88 \%$ accuracy. In a study of 100 sleep-disordered patients, Kushida et al. [5] reported that total sleep time and sleep efficiency results that did not differ significantly from those obtained by PSG data were obtained by a combination of actigraphy and subjective reports. Long et al. [6] reported 95.7\% accuracy and a kappa of 0.59 for a sleep-wakefulness classification by a combination of actigraphy and respiration in a study of 15 healthy subjects. Additionally, Aktaruzzaman et al. [9] have recently reported classification performance by a combination of actigraphy and HRV in 18 subjects with no previous history of sleep disorders. They found that sleep and waking were distinguished at $78 \%$ accuracy by four features derived only from wrist actigraphy, and that the addition of HRV features resulted in no significant improvement of classification performance. Although there is an apparent discrepancy between their findings and those in the present study, they reported about sleep-wakefulness classification in subjects without sleep disorder, while we performed sleep stage classification in subjects consisting mainly $(88 \%)$ of patients with sleep disorders.

The present study is characterized by: (1) providing sleep stage classification models with the combined use of actigraphic and HR data; (2) proposing two-step approaches separately discriminating NREM sleep from waking/REM sleep and REM sleep from wakefulness; and (3) examining models with a large sample size. Our observations support the merit of the combined use of HR and actigraphic data for the estimation of sleep stage.

This study has limitations. First, in this study, 30.5\% of 3-min epochs were defined as a transient stage and were excluded from the analysis. The definition of transient stage, however, could affect the performance of the classification. Second, we used only two-way classification accuracies: NREM from REM and waking stages and REM from the waking stage. This is because we tried to characterize the parameters contributing to each classification in the physiological point of view. To determine the models' practical usefulness, however, performance for three-way classification is important. Finally, the classification of sleep stage may be affected by sleep-disordered breathing. Particularly, because episodes of sleep apnea are accompanied by a cyclic variation of heart rate [16,17], further studies are required for the impact of sleep-disordered breathing on sleep stage classification by HRV indices.

\section{Conclusions}

We demonstrated the performance of sleep stage classification by the indices obtained from HR and actigraphic signals. In the analysis of 40,643 3-min epochs of PSG data in 289 subjects, NREM was discriminated from the waking/REM stages by the combination of HF, VLF, HR, and BM indices with $75.8 \%$ accuracy and a kappa $=0.514$, and REM sleep was discriminated from the waking stage by the combination of VLF, HR, and BM indices with $74.5 \%$ accuracy and kappa $=0.491$. Our observations indicate that the combined use of autonomic functional indices from HRV and BM indices derived 
from actigraphic data are useful for discriminating NREM from the REM/waking stages and for discriminating REM from the waking stage.

Author Contributions: H.T., T.S. and J.H. conceived and designed the study; R.S. and H.T. performed the experiments; Y.Y. and J.H. developed computer algorithms; E.Y., Y.Y. and J.H. analyzed the data; and E.Y. and J.H. wrote the paper.

Conflicts of Interest: The authors declare no conflict of interest.

\section{References}

1. Penzel, T.; Kantelhardt, J.W.; Grote, L.; Peter, J.H.; Bunde, A. Comparison of detrended fluctuation analysis and spectral analysis for heart rate variability in sleep and sleep apnea. IEEE Trans. Biomed. Eng. 2003, 50, 1143-1151. [CrossRef] [PubMed]

2. Adane, M.; Jiang, Z.; Yan, Z. Sleep-wake stages classification and sleep efficiency estimation using single-lead electrocardiogram. Expert Syst. Appl. 2012, 39, 1401-1413. [CrossRef]

3. Fonseca, P.; Long, X.; Radha, M.; Haakma, R.; Aarts, R.M.; Rolink, J. Sleep stage classification with ECG and respiratory effort. Physiol. Meas. 2015, 36, 2027-2040. [CrossRef] [PubMed]

4. Cole, R.J.; Kripke, D.F.; Gruen, W.; Mullaney, D.J.; Gillin, J.C. Automatic sleep/wake identification from wrist activity. Sleep 1992, 15, 461-469. [CrossRef] [PubMed]

5. Kushida, C.A.; Chang, A.; Gadkary, C.; Guilleminault, C.; Carrillo, O.; Dement, W.C. Comparison of actigraphic, polysomnographic, and subjective assessment of sleep parameters in sleep-disordered patients. Sleep Med. 2001, 2, 389-396. [CrossRef]

6. Long, X.; Fonseca, P.; Foussier, J.; Haakma, R.; Aarts, R.M. Sleep and wake classification with actigraphy and respiratory effort using dynamic warping. IEEE J. Biomed. Health Inform. 2014, 18, 1272-1284. [CrossRef] [PubMed]

7. Nakazaki, K.; Kitamura, S.; Motomura, Y.; Hida, A.; Kamei, Y.; Miura, N.; Mishima, K. Validity of an algorithm for determining sleep/wake states using a new actigraph. J. Physiol. Anthropol. 2014, 33. [CrossRef] [PubMed]

8. Aktaruzzaman, M.; Migliorini, M.; Tenhunen, M.; Himanen, S.L.; Bianchi, A.M.; Sassi, R. The addition of entropy-based regularity parameters improves sleep stage classification based on heart rate variability. Med. Biol. Eng. Comput. 2015, 53, 415-425. [CrossRef] [PubMed]

9. Aktaruzzaman, M.; Rivolta, M.W.; Karmacharya, R.; Scarabottolo, N.; Pugnetti, L.; Garegnani, M.; Bovi, G.; Scalera, G.; Ferrarin, M.; Sassi, R.; et al. Performance comparison between wrist and chest actigraphy in combination with heart rate variability for sleep classification. Comput. Biol. Med. 2017, 89, 212-221. [CrossRef] [PubMed]

10. Bonnet, M.H.; Arand, D.L. Heart rate variability: Sleep stage, time of night, and arousal influences. Electroencephalogr. Clin. Neurophysiol. 1997, 102, 390-396. [CrossRef]

11. Vanoli, E.; Adamson, P.B.; Ba, L.; Pinna, G.D.; Lazzara, R.; Orr, W.C. Heart rate variability during specific sleep stages: A comparison of healthy subjects with patients after myocardial infarction. Circulation 1995, 91, 1918-1922. [CrossRef] [PubMed]

12. Elsenbruch, S.; Harnish, M.J.; Orr, W.C. Heart rate variability during waking and sleep in healthy males and females. Sleep 1999, 22, 1067-1071. [CrossRef] [PubMed]

13. Villa, M.P.; Calcagnini, G.; Pagani, J.; Paggi, B.; Massa, F.; Ronchetti, R. Effects of sleep stage and age on short-term heart rate variability during sleep in healthy infants and children. Chest 2000, 117, 460-466. [CrossRef] [PubMed]

14. Hayano, J. Introduction to heart rate variability. In Clinical Assessment of the Autonomic Nervous System; Iwase, S., Hayano, J., Orimo, S., Eds.; Springer: Tokyo, Japan, 2016; pp. 109-127.

15. Camm, A.J.; Malik, M.; Bigger, J.T., Jr.; Breithardt, G.; Cerutti, S.; Cohen, R.J.; Coumel, P.; Fallen, E.L.; Kennedy, H.L.; Kleiger, R.E.; et al. Task Force of the European Society of Cardiology and the North American Society of Pacing and Electrophysiology. Heart rate variability: Standards of measurement, physiological interpretation and clinical use. Circulation 1996, 93, 1043-1065. 
16. Guilleminault, C.; Connolly, S.; Winkle, R.; Melvin, K.; Tilkian, A. Cyclical variation of the heart rate in sleep apnoea syndrome. Mechanisms, and usefulness of $24 \mathrm{~h}$ electrocardiography as a screening technique. Lancet 1984, 1, 126-131. [CrossRef]

17. Hayano, J.; Watanabe, E.; Saito, Y.; Sasaki, F.; Fujimoto, K.; Nomiyama, T.; Kawai, K.; Kodama, I.; Sakakibara, H. Screening for obstructive sleep apnea by cyclic variation of heart rate. Circ. Arrhythm. Electrophysiol. 2011, 4, 64-72. [CrossRef] [PubMed] 